\title{
Privacy-by-Design Distributed Offloading for Vehicular Edge Computing
}

\author{
Weibin Ma \\ University of Delaware \\ Department of Computer and \\ Information Sciences \\ Newark, DE, USA \\ weibinma@udel.edu
}

\author{
Lena Mashayekhy \\ University of Delaware \\ Department of Computer and \\ Information Sciences \\ Newark, DE, USA \\ mlena@udel.edu
}

\begin{abstract}
Vehicular Edge Computing (VEC) is a distributed computing paradigm that utilizes smart vehicles (SVs) as computational cloudlets (edge nodes) by virtue of their inherent attributes such as mobility, low operating costs, flexible deployment, and wireless communication ability. VEC extends edge computing services by expanding computing coverage and further improving quality-of-services (QoS) for devices. Due to limited onboard energy and computation capabilities of SV-mounted cloudlets, a single vehicle might not be able to execute a large number of tasks and guarantee their desired QoS. To address this problem, the overloaded vehicle can fulfill its overwhelming workload by offloading its tasks to other available connected vehicles. However, data privacy and accessibility are of critical importance that need to be considered for offloading. In this paper, we propose privacy-by-design offloading solutions for VEC to facilitate latency requirements of user demands and reduce energy consumption of vehicles. We formulate the Data pRotection Offloading Problem (DROP) as an Integer Program and prove its NP-hardness. To provide computationally tractable solutions, we propose three distributed algorithms by leveraging graph theory to solve this problem. We evaluate the performance of our proposed algorithms by extensive experiments and compare them to the optimal results obtained by IBM ILOG CPLEX. The results demonstrate the flexibility, scalability, and cost efficiency of our proposed algorithms in providing practical privacy-by-design offloading solutions enabling edge services along the cloud-to-thing continuum.
\end{abstract}

\section{KEYWORDS}

Vehicular Edge Computing, Smart Connected Vehicles, Internet-ofThings, Data Protection, Distributed Algorithms

\section{ACM Reference Format:}

Weibin Ma and Lena Mashayekhy. 2019. Privacy-by-Design Distributed Offloading for Vehicular Edge Computing. In IEEE/ACM 12th International Conference on Utility and Cloud Computing (UCC'19), December 2-5, 2019, Auckland, New Zealand. ACM, New York, NY, USA, 10 pages. https://doi. org $/ 10.1145 / 3344341.3368804$

Permission to make digital or hard copies of all or part of this work for personal or classroom use is granted without fee provided that copies are not made or distributed for profit or commercial advantage and that copies bear this notice and the full citation on the first page. Copyrights for components of this work owned by others than ACM must be honored. Abstracting with credit is permitted. To copy otherwise, or republish, to post on servers or to redistribute to lists, requires prior specific permission and/or a fee. Request permissions from permissions@acm.org.

UCC '19, December 2-5, 2019, Auckland, New Zealand

(C) 2019 Association for Computing Machinery.

ACM ISBN 978-1-4503-6894-0/19/12 . \$ 15.00

https://doi.org/10.1145/3344341.3368804

\section{INTRODUCTION}

The ubiquitous penetration of smart connected devices (Internet of Things) into everyday life is projected to reach 50 billion by 2020 [23]. The growth of IoT will continue as users enjoy the convenience of mobility and with the emergence and progress of new technologies such as wearable devices, autonomous vehicles/drones, and collaborative augmented/virtual reality. To enable these IoT applications and scale over the number of participants and large geographical areas, computational capabilities of IoT devices are not sufficient due to being restricted by weight, size, battery life, and heat dissipation. To handle this challenge, offloading computation to clouds to remotely execute IoT applications is one of the promising solutions. As data proliferation increases exponentially, however, sending data from IoT devices to the cloud is not feasible for time-sensitive applications.

Edge Computing (EC) has been introduced as a new paradigm [21] that optimizes cloud computing systems to provide a distributed computing solution at the edge of the network, where IoT devices utilize the computing resources, called cloudlets, in their vicinity. Edge computing can be leveraged to bridge the gap between the increasing computational demand of IoT devices and their limited computational capabilities [24]. However, deploying cloudlet infrastructure at the edge of the network is costly and may not be feasible in many situations (e.g., disaster situations, emergency rescue, unexpected surge in user demand) and regions with sparse or no infrastructure of wireless access points such as remote rural areas [1]. Moreover, a single cloudlet has finite computing resources, which makes it hard to fulfill demand spikes (e.g., a massive number of offloading requests from IoT devices).

To overcome these problems, Vehicular Edge Computing (VEC) has recently been introduced as an emerging edge platform [4, 16, 17, 30], where smart vehicles (SVs) such as Unmanned Aerial Vehicles (UAVs) and Connected and Autonomous Vehicles (CAVs) are considered as computational cloudlets by virtue of their inherent attributes such as mobility, low operating costs, flexible deployment, and wireless communication ability. SV-mounted cloudlets can expand edge computing services and further improve quality-ofservices (QoS) for IoT devices.

While VEC can bring many opportunities to avoid QoS violations and balance the load, these SV-mounted cloudlets have often limited computing capacities and energy budgets. To expand the computational capacity and coverage of a single SV-mounted cloudlet, especially when serving large computational demands, autonomous cooperation and coordination among multiple SVs are needed to form an interconnected computing system and improve 
quality of edge services $[11,12,22]$. Such a perspective, on the other hand, opens new research challenges on account of the current lack of efficient data protection mechanisms for task offloading. In particular, the major categories are considered privacy and accessibility restrictions defined as follows:

Privacy restrictions: If some tasks are offloaded to the same SV, they can reveal sensitive information, which could harm IoT user privacy $[15,18]$ (e.g., in finance and healthcare data).

Accessibility restrictions: There are often some restrictions in offloading a task to a specific SV due to lack of trust, reliability, or system compatibility/preferences [14, 27] (e.g., cannot provide a proper type of VM for a task). In addition, there may exist some enforced policies that do not allow a third party to access confidential user data.

These restrictions eliminate the privacy-breach problem and enable privacy-by-design solutions. Many studies have been conducted to protect outsourced data by designing steganography and encryption [9]. However, these algorithms have limitations, especially in edge computing domain, due to requiring additional processing before offloading. Moreover, encryption may dramatically increase the amount of data. Privacy by design is a suitable approach for offloading in VEC, by incorporating privacy principles as early as in the design phase of systems-a proactive rather than reactive to risk.

In this paper, we consider a set of SV-mounted cloudlets cooperating to provide edge computing services for IoT devices. They are capable of both communicating with the devices and offloading computation to other SVs via the wireless communication technology in order to fulfill the overwhelming demand. We focus on the critical problem of offloading tasks from an overloaded SV to minimize computation overhead in terms of energy consumption and processing time while satisfying privacy and accessibility restrictions. We first design an optimal offloading mathematical model for this Data pRotection Offloading Problem (DROP), and we theoretically prove that this is an NP-hard problem. We then propose three distributed algorithms by virtue of graph theory to obtain efficient and computationally tractable solutions in order to minimize the total computation overhead of the offloading. We finally evaluate the performance of our proposed solutions in extensive experiments. To the best of our knowledge, this is the first work that provides privacy-by-design offloading solutions among a set of cooperative SVs.

Organization. The rest of the paper is organized as follows. In Section 2, we discuss the state-of-the-art research in this domain. In Section 3, we introduce the problem of optimal task offloading among cooperative SVs considering the privacy and accessibility restrictions, and we mathematically formulate the problem. In Section 4, we present our proposed computationally tractable algorithms. In Section 5, we evaluate our proposed algorithms by extensive experiments. In Section 6, we summarize our results and present possible directions for future research.

\section{RELATED WORK}

The key challenges of DROP lie in the combinatorial nature of offloading decisions, the necessity of data protection requirements, and the limited capacities of SVs. Due to the dynamics and unplanned deployment of SV-mounted cloudlets, centralized optimization approaches for task offloading may not be efficient in such a distributed environment. Moreover, they require each IoT user to report his/her own information including the capacity of the IoT device and the size of each task to a centralized entity, e.g., cloud, which decides the offloading decisions accordingly. Therefore, extra concerns such as high computational complexity, tremendous communication overhead, and massive data transfers, are unavoidable in centralized optimization. All these points accelerate the emergence of efficient offloading schemes by designing decentralized approaches.

Recently, a few studies $[12,13,16]$, devised distributed approaches to optimize the offloading problem in EC by exploiting multi-player noncooperative games. Messous et al. [16] presented a gametheoretic approach to address the intensive computation offloading problem for multiple UAVs, and they showed that the formulated game admits a Nash equilibrium. Ma et al. [12] investigated the feasibility of offloading computational tasks in a network of capacitated UAV-mounted cloudlets in order to minimize the energy consumption of UAVs while satisfying QoS requirements of the tasks. They devised an efficient decentralized approach based on potential games. Ma et al. [13] proposed a distributed computation offloading approach for multiple users to offload their tasks to a single cloudlet via multiple access points. However, the game theoretic approaches present some drawbacks [5]. For example, finding the best response of each player usually requires the knowledge of other players' actions at each round, which instead increase the computational complexity of the solution. Furthermore, to find an equilibrium of a game, the objective function requires a specific structure.

With the rapid proliferation of smart vehicles, the task offloading problem in VEC has received considerable attention. Zhang et al. [29] proposed a contract-based offloading and computation resource allocation scheme in VEC. Zhang et al. in [28] studied the feasibility of combining vehicular cloudlets with the centralized cloud and proposed a flexible offloading strategy to explore underutilized resources via task migration. Yu et al. in [26] proposed a coalitional game model for cooperation among cloud service providers in cloud-enabled vehicular networks in order to share and utilize idle resources.

Nevertheless, none of the existing work investigated both data privacy and cloudlets accessibility in a multi-cloudlet cooperative computing system.

\section{PRIVACY-BY-DESIGN OPTIMIZATION FORMULATION}

In this section, we introduce the system model and the data protection offloading problem.

\subsection{System Model}

In this subsection, we describe the system model with a set of SVs acting as mobile computational cloudlets that provide edge computing services to IoT devices. We consider that an overloaded SV has to offload its overwhelming computation to other SVs in order to fulfill its demands and guarantee the desired QoS of its workload. 
The objective is to minimize the overhead cost of offloading while satisfying the aforementioned privacy and accessibility restrictions. This is an offloading decision-making problem that requires an efficient and proactive data protection offloading solution.

We consider $u_{0}$ as the overloaded SV and $U=\left\{u_{1}, \ldots, u_{m}\right\}$ as the set of $m$ SVs with available computational resources. The set of tasks at SV $u_{0}$ to be offloaded is denoted by $K=\left\{k_{1}, \ldots, k_{n}\right\}$, where $n \geq 1$ is the number of tasks ( $u_{0}$ has at least one task to be offloaded). Let $F_{i}$ be the CPU frequency (i.e., CPU cycles per second) of $u_{i}$, and $e_{i}$ denotes its energy consumption per CPU cycle. The capacity of $u_{i}$ is denoted by $u_{i}^{c a p}=\left(C_{i}^{c a p}, D_{i}^{c a p}\right)$, where $C_{i}^{c a p}$ is the number of idle computational cycles of $u_{i}$ and $D_{i}^{c a p}$ is the available memory size of $u_{i}$. Each task $k_{i} \in K$ has some requirements defined by $\left(C_{i}, D_{i}\right)$, where $C_{i}$ represents the number of computational cycles required to obtain the outcome of $k_{i}$ and $D_{i}$ denotes its data size. Similar to previous studies in mobile wireless networks [2,7], for tractability, we assume that the locations of SVs remain fixed during the offloading decision making. More details are presented in the following subsections.

SV $u_{0}$ does not have enough computational resources to complete all its incoming tasks with their required QoS, and hence this overloaded SV has to offload these tasks to other SVs. As we described in the Introduction Section, there are two restrictions to be considered when offloading tasks to other SVs: (i) the privacy restrictions, specifying that some tasks cannot be offloaded to the same SV; (ii) the accessibility restrictions, specifying that a task cannot be offloaded to a specific SV. To model these two restrictions, we visualize them in the form of a conflict graph and searching matrix, respectively.

We model the privacy restrictions using a conflict graph $\mathcal{G}\langle\mathbf{V}$, $\mathbf{E}\rangle$, where $\mathbf{V}=\left\{v_{1}, \ldots, v_{n}\right\}$ is a set of vertices representing the tasks in $K^{1}$ and $\mathbf{E}$ is a set of edges $\langle i, j\rangle$ representing a conflict between task $k_{i}$ and $k_{j}$, where $i, j \in\{1, \ldots, n\}$. Specifically, if tasks $k_{i}$ and $k_{j}$ cannot be offloaded to the same SV, an edge $\langle i, j\rangle$ is

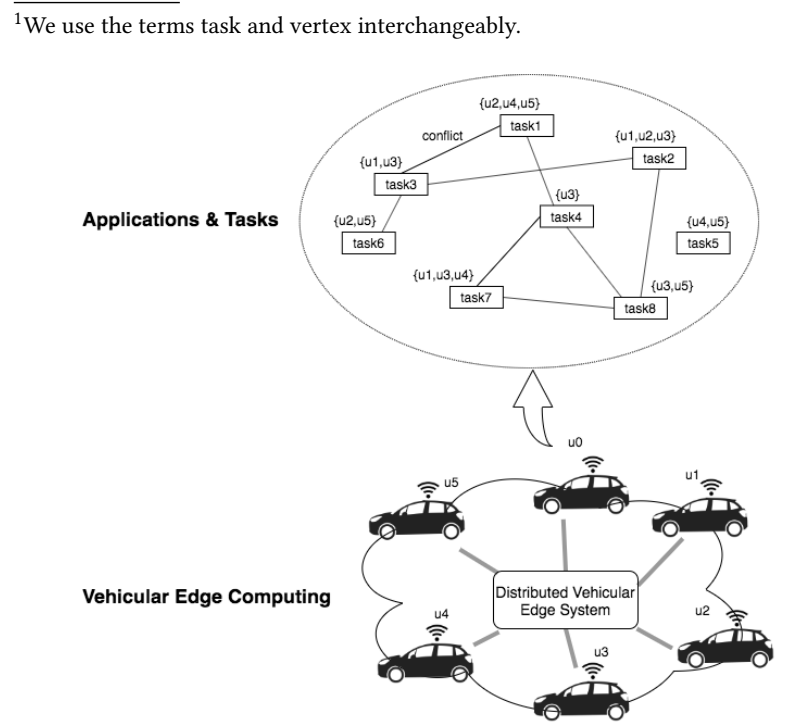

Figure 1: VEC architecture: an overloaded SV $u_{0}$ offloads its tasks to other SVs added between $v_{i}$ and $v_{j}$ in the graph. If there is no edge between two vertices, their corresponding tasks can be offloaded to the same $\mathrm{SV}$. Therefore, $\mathcal{G}$ is an undirected graph, and $\mathbf{E}$ is symmetric.

We model the accessibility restrictions by a $m \times n$ matrix $\mathcal{T}_{m, n}=$ $\left(t_{i j}\right)$, where the $i$ th row represents SV $u_{i} \in U$ and the $j$ th column represents task $k_{j} \in K$. In particular, if SV $u_{i}$ is accessible to task $k_{j}$, we set $t_{i j}=1$; otherwise, $t_{i j}=0$.

Fig 1 shows an overloaded SV offloads its overwhelming tasks to other SVs with enough computational resources via vehicle-tovehicle $(\mathrm{V} 2 \mathrm{~V})$ communications, while considering the data protection restrictions.

\subsection{Data Protection Offloading Problem}

In this subsection, we introduce the optimal mathematical model for the data protection offloading problem. We first describe the communication, energy consumption, and latency models, and we then present our optimization model.

1) Communication Model.

To avoid generating severe interference during offloading tasks, we consider the overloaded SV applies a Frequency-Division Multiple Access scheme (FDMA) to transmit tasks to other SVs. In FDMA, each channel between $u_{0}$ and $u_{j} \in U$ is unique, and $u_{0}$ can hence offload tasks to other SVs simutaneously without co-channel interference. As a result, the transmission rate of $u_{0}$ to $u_{j}$ is defined as [20]:

$$
R_{0 j}=B_{j} \log \left(1+\frac{P_{0}}{N_{0} d_{0 j}^{h}}\right),
$$

where $B_{j}$ is the total bandwidth of $u_{j}, P_{0}$ denotes the transmission power of $u_{0}$, and $d_{0 j}$ is the distance between $u_{0}$ and $u_{j}$. In addition, $N_{0}$ and $h$ represent the background noise and the path-loss factor, respectively.

\section{2) Energy Consumption Model.}

The total energy consumption for completing a task via offloading (e.g., from $u_{0}$ to $u_{j}$, where $u_{j} \in U$ ) consists of three components: the transmission energy consumption from $u_{0}$ to $u_{j}$, the execution energy consumption at $u_{j}$, and the backhaul energy consumption of the outcomes of computation from $u_{j}$ to $u_{0}$. Similar to many studie $[2,6,12]$, the backhaul energy consumption can be ignored, since the size of output is generally much smaller than the size of input. As a result, the total energy consumption for completing task $k_{i}$ via offloading from $u_{0}$ to $u_{j}$ is calculated as:

$$
E_{j}(i)=\frac{D_{i} P_{0}}{R_{0 j}}+C_{i} e_{j} .
$$

The first term represents the transmission energy, and the second term represents the energy consumption of executing the task at $u_{j}$.

3) Latency Model.

Likewise, the total delay of completing task $k_{i}$ by offloading (from $u_{0}$ to $u_{j}$ ) is calculated as:

$$
T_{j}(i)=\frac{D_{i}}{R_{0 j}}+\frac{C_{i}}{F_{j}},
$$


where the first term represents the total transmission time of $k_{i}$ from $u_{0}$ to $u_{j}$, and the second term represents the total execution time of $k_{i}$ at $u_{j}$.

\section{4) Optimization Model.}

Both latency and energy consumption are important factors in offloading among SVs. One of the main limitations of SVs is their restricted battery lifetime. On the other hand, most IoT applications are sensitive to delay such as video streaming and real-time games. We hence consider an overhead cost as a combination of both latency and energy consumption. According to (1) and (2), the overhead cost of offloading task $k_{i}$ to $u_{j}$ in terms of the energy and latency is calculated by:

$$
Z_{j}(i)=\alpha_{i} E_{j}(i)+\beta_{i} T_{j}(i),
$$

where $0 \leq \alpha_{i}, \beta_{i} \leq 1$ represent the relative weights of energy consumption and latency of the objectives, and $\alpha_{i}+\beta_{i}=1$. This provides rich modeling flexibility to prioritize the objectives (energy and latency) according to the required QoS of the applications and the current battery status of the SVs. For instance, when a task is delay-sensitive and the SVs are at a high-battery state, more weight is assigned to the latency (i.e., higher $\beta_{i}$ ). In practice, the proper weights can be determined by exploiting multiple criteria decision making (MCDM) and multi-attribute utility theory (MAUT) approaches [25].

We define an indicator variable $b_{i j}, \forall i \in\{1, \ldots, n\}, \forall j \in\{1, \ldots, m\}$, that characterizes the accessibility relationship between task $k_{i}$ and SV $u_{j}$ as follows:

$$
b_{i j}= \begin{cases}1 & \text { if task } k_{i} \text { can be offloaded to } u_{j} \\ 0 & \text { otherwise }\end{cases}
$$

This indicates whether SV $u_{j}$ is accessible to task $k_{i}$ or not. We then define a decision variable $x_{i j}$ as follows:

$$
x_{i j}= \begin{cases}1 & \text { if task } k_{i} \text { is offloaded to } u_{j}, \\ 0 & \text { otherwise. }\end{cases}
$$

This specifies whether task $k_{i}$ is offloaded to SV $u_{j}$ or not. We now formulate the data protection offloading problem (DROP) as an Integer Program (IP), called IP-DROP, as follows:

$$
\text { Minimize } \mathcal{Z}=\sum_{i=1}^{n} \sum_{j=1}^{m} Z_{j}(i) x_{i j}
$$

Subject to:

$$
\begin{array}{lr}
\sum_{i=1}^{n} C_{i} x_{i j} \leq C_{j}^{c a p}, & \forall j \in\{1, \ldots, m\}, \\
\sum_{i=1}^{n} D_{i} x_{i j} \leq D_{j}^{c a p}, & \forall j \in\{1, \ldots, m\}, \\
\sum_{j=1}^{m} b_{i j} x_{i j}=1, & \forall i \in\{1, \ldots, n\}, \\
x_{v j}+x_{v^{\prime} j} \leq 1, & \forall\left\langle v, v^{\prime}\right\rangle \in \mathbf{E}, \forall j \in\{1, \ldots, m\}, \\
x_{i j} \in\{0,1\}, & \forall i \in\{1, \ldots, n\}, \forall j \in\{1, \ldots, m\} .
\end{array}
$$

The objective function (4) is to minimize the overhead cost of offloading all tasks in $K$. Constraints (5)-(6) ensure that the assignment of the offloaded tasks to each SV does not exceed the available computational cycles and memory size of that SV, respectively. Constraints (7) ensure that each task is offloaded to exactly one SV obeying the accessibility restrictions. Constraints (8) ensure the privacy restrictions such that any two conflicted tasks cannot be offloaded to the same SV. Finally, Constraints (9) guarantee that the decision variables are binary.

\subsection{Computational Complexity of DROP}

To verify the computational complexity of DROP, we first introduce the related preliminaries from graph theory.

Definition 1. Given a graph $G(V, E)$ and a set of colors, a proper coloring is an assignment of colors to vertices such that no two adjacent vertices have the same color.

Definition 2. Given a graph $G(V, E)$ and a list of colors $L(v)$ for $v \in V$, a proper list coloring is a choice function $c(\cdot)$ that maps every vertex $v \in V$ to a color in the list $L(v)$ such that $c(v) \in L(v)$ and $c(i) \neq c(j)$ if $\langle i, j\rangle \in E$ for all $i, j \in V$.

We now define DROP as a list coloring problem (LCP) considering $\mathcal{G}(\mathbf{V}, \mathbf{E})$ and $\mathcal{T}_{m, n}$. Each task $v \in \mathbf{V}$, represented by a vertex, is given a list of accessible SVs (permissible colors) ${ }^{2}$ based on $\mathcal{T}_{m, n}$. For our analysis, we let each task $v \in \mathbf{V}$ have a list of permissible SVs $L(v) \subseteq\left\{u_{1}, u_{2}, \ldots, u_{m}\right\}$, where $m$ is the total number of SVs. We then sort SVs in $L(v)$ based on their corresponding overhead cost in ascending order. We define the sorted list of permissible SVs of task $v$ as $L^{s}(v)$ and its corresponding sorted overhead cost list is defined as $Z^{s}(v)$.

Lemma 1. If all tasks have the same requirements (computation $C_{i}$ and data size $D_{i}$, for all tasks $k_{i} \in K$ ) and considering consistent priorities for the objectives $\left(\alpha_{i}, \beta_{i}\right.$ remains the same), then the offloading cost $Z_{j}(i)$ to any $u_{j}$ is the same for all tasks $k_{i} \in K$.

Proof. Since the offloading scenario in DROP is that an overloaded SV $u_{0}$ offloads $n$ tasks $K=\left\{k_{1}, \ldots, k_{n}\right\}$ via FDMA scheme to $m$ SVs, the parameters $P_{0}, N_{0}, h, \gamma, B_{j}, F_{j}, d_{0 j}$ with respect to any $\mathrm{SV} u_{j}$ are hence constant. Therefore, if any two tasks $k_{u}, k_{w} \in K$ have the same requirements $C_{u}=C_{w}, D_{u}=D_{w}$ while $\alpha_{u}=$ $\alpha_{w}, \beta_{u}=\beta_{w}$, according to Eq. (1-3), for these tasks we have $Z_{j}(u)=Z_{j}(w), \forall j \in\{1, \ldots, m\}$.

In graph theory, a sum coloring of a graph is a labeling of its vertices by natural numbers (positive integers), with no two adjacent vertices having equal labels, that minimizes the sum of the labels. The minimum sum that can be achieved is called the chromatic sum of the graph.

Definition 3. The chromatic sum $\sum G$ of graph $G$ is the smallest sum of labels (colors) among all proper colorings with natural numbers.

The chromatic sum problem is NP-complete [10]. We now prove the computational complexity of DROP. The following lemma constructs the decision version of DROP by imposing a bound on the cost value and proves its NP-completeness.

\footnotetext{
${ }^{2}$ From now, we use the terms SV and color interchangeably.
} 
Lemma 2. The decision version of DROP, called D-DROP, is NPcomplete: Given $\mathcal{G}\langle\mathbf{V}, \mathbf{E}\rangle, \mathcal{T}_{m, n}$, and a value $F$, is there a complete assignment such that the overhead cost of all tasks, called $\mathcal{Z}$, does not exceed $F$ (i.e., $\mathcal{Z} \leq F$ )?

Proof. The first step is to prove that D-DROP is in NP by showing that given a complete assignment $\mathcal{X}$, it can be decided in polynomial time that $\mathcal{X}$ is a solution to the problem or not. This is easy to show since a nondeterministic program guesses a complete assignment $\mathcal{X}$ for all vertices and checks it in polynomial time whether this assignment is feasible and $\mathcal{Z} \leq F$.

The second step is to find a polynomial-time reduction from the decision version of the chromatic sum problem (denoted here by CSP-D), a well known NP-complete problem [10], to D-DROP. The CSP-D problem is defined as follows: Given graph $G(V, E)$ and an integer $F$, is there a feasible coloring $c$ of graph $G$ such that $\sum_{v \in V} c(v) \leq F$ ? We construct an instance of D-DROP as follows: We use a one-to-one mapping between $\mathcal{G}(\mathbf{V}, \mathbf{E})$ and $G(V, E)$, and considering $F$. We let each task $v \in V$ have a list of permissible SVs $L(v)=\left\{u_{1}, u_{2}, \ldots, u_{m}\right\}$, where $m$ is the total number of SVs. Following Lemma 1, we assume all tasks $k_{i} \in K$ have the same required computational cycles and data size for simplicity. Therefore, all tasks have the same $L^{s}(v)$ and $Z^{s}(v)$. The Yes/No answer to the D-DROP instance corresponds to the same answer as for the CSP-D instance. Is there a feasible complete assignment $\mathcal{L}=\{l(v) \mid l(v) \in L(v)\}$ such that $\sum_{v \in V} Z_{l(v)}(v) \leq F$, where $Z_{l(v)}(v) \in Z^{s}(v)$ is the overhead cost that task $v$ is offloaded to its permissible SV $l(v)$ ? Obviously, the new constructed problem is equivalent to the CSP-D, and this construction is done in polynomial time. Therefore, we have the CSP-D $\leq_{P} \mathrm{D}$-DROP. In other words, we can transform the decision version of the chromatic sum problem to a special case of D-DROP in polynomial time.

Therefore, the D-DROP is NP-complete.

The decision version of a problem is easier than (or the same as) the optimization version. We now prove the computational complexity of the optimization version of DROP.

Theorem 1. The optimization version of $D R O P(O-D R O P)$ for finding the minimum overhead cost of offloading all tasks is NP-hard.

Proof. The proof is by contradiction. If a polynomial-time algorithm can be found to solve O-DROP, it implies that we can obtain $\mathcal{Z}$ in polynomial time. Then, we just need to check if there is a solution for D-DROP considering $\mathcal{Z}$ as $F$. Obviously, this comparison is done in polynomial time, and thus, the D-DROP can be solved in polynomial time, which contradicts Lemma 2. Since the D-DROP is NP-complete, then the O-DROP is NP-hard (D-DROP $\leq_{P}$ O-DROP).

\section{DISTRIBUTED SOLUTIONS}

In this section, we propose three algorithms for solving DROP. The reason we prefer distributed algorithms is due to their robustness and scalability. Since DROP is NP-hard, optimal solutions may only be obtained when the problem size is relatively small (a few vehicles and tasks). We design proper distributed offloading algorithms that

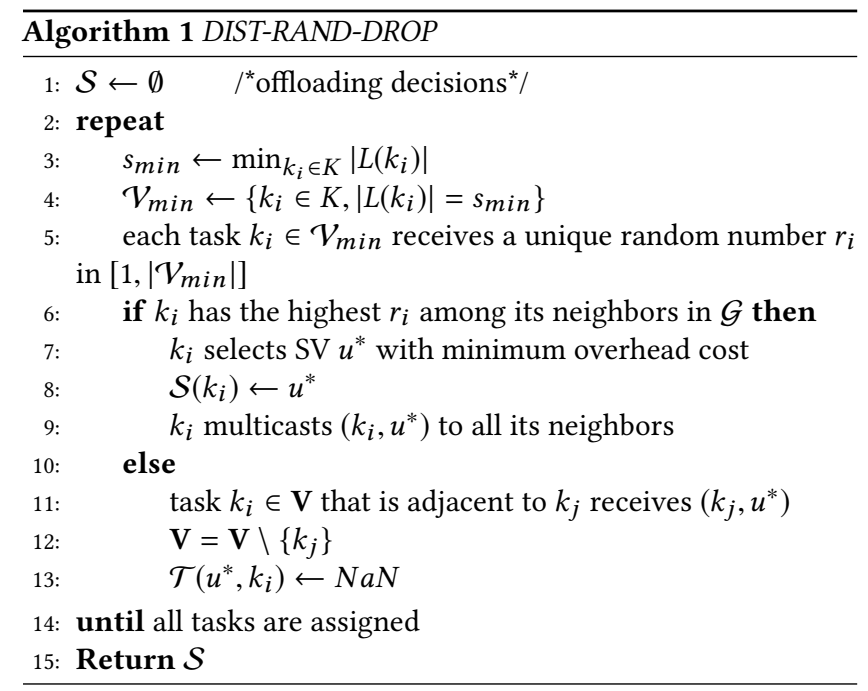

are computationally tractable in finding complete assignments with small overhead cost for privacy-by-design task offloading.

\subsection{Distributed Randomized Algorithm}

In this subsection, we design an iterative randomized distributed algorithm called DIST-RAND-DROP in which the offloading will be based on a uniform distribution. In each round, tasks with the highest value among their neighbors (i.e., conflicting tasks) select their SVs for offloading. Then, each of these tasks, called a winning task, multicasts its offloading information to all its neighbors in which the selected SV will be removed from their permissible list. Next, these winning tasks are removed, and the remaining tasks that lose this contention update their own sets for the next round.

DIST-RAND-DROP, given in Algorithm 1, works as follows. It defines integer $s_{\min }$ to be the minimum number of permissible SVs for any task $k_{i} \in K$. Then, a list of tasks with minimum number of permissible SVs, $s_{\text {min }}$, is added to $\mathcal{V}_{\text {min }}$. Each task in $\mathcal{V}_{\text {min }}$ receives a unique random number uniformly from $\left[1,\left|\mathcal{V}_{\text {min }}\right|\right]$ without any replacement. Within one round, if one task has the highest random number among all its neighbors, it wins the contention and selects its permissible SV with minimum overhead cost. Then each winning task multicasts offloading information to all its neighbors in which the selected SV will be removed from their permissible SVs lists (by setting it to $N a N$ or not available). Next, these winning tasks are removed from $\mathbf{V}$ and the remaining tasks that lose the contention update their own $\mathcal{U}^{c a p}, \mathcal{G}(\mathbf{V}, \mathbf{E}), \mathcal{T}(m, n)$. The algorithm continues to the next round.

Example 1 (Infeasible assignment). This algorithm can result in infeasible assignments. Consider a privacy-restriction conflict graph shown in Fig. 2, where all tasks have the same size of permissible SVs list (here 2) and all SVs have adequate capacity. According to the property of DIST-RAND-DROP, a unique random number is assigned to each task (shown in green). Task 2 has the highest number among all its neighbors, thus it selects the first SV in its list (i.e., $u_{1}$ ) and multicasts it to other tasks to update their lists for the next rounds. This selection results in an infeasible assignment, since both tasks 3 and 4 have only SV $u_{3}$ for offloading 


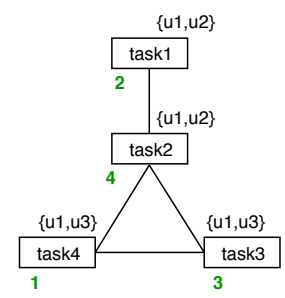

Figure 2: Infeasible assignment

while they conflict with each other and cannot be offloaded to the same SV.

This encourages us to design an efficient selection function at each round to improve the probability of finding a complete assignment.

\subsection{Distributed Clique Algorithm}

In this subsection, we introduce our second algorithm called DISTCLIQUE-DROP, shown in Algorithm 2, that considers feasibility of assignments in cliques.

Given the conflict graph $\mathcal{G}(\mathbf{V}, \mathbf{E})$, accessibility matrix $\mathcal{T}_{m, n}$, and SVs capacity $\mathcal{U}^{\text {cap }}$, the algorithm first calculates $s_{\text {min }}$ and $\mathcal{V}_{\text {min }}$. It then arbitrarily selects a task $k_{i} \in \mathcal{V}_{\text {min }}$, and sorts its permissible SVs in ascending order of their overhead cost, denoted by $L^{s}\left(k_{i}\right)$. Next, this task checks whether a selected SV from the list $L^{s}\left(k_{i}\right)$ leads to a feasible assignment. This step is done using cliques. First, all cliques containing any adjacent task $k_{j} \in \mathbf{V}$ to $k_{i}$, where $\langle i, j\rangle \in$ $\mathbf{E}$ are found. If the number of unique SVs in each of these cliques is larger than or equal to the clique size itself, then there is a feasible assignment. Task $k_{i}$ investigates if choosing the first SV from the list $L^{s}\left(k_{i}\right)$ (minimum cost) leads to a feasible assignment for all neighboring tasks. Otherwise, it chooses the next SV in the list and check the feasibility. This is a backtracking search approach, and it is halted when all the neighboring tasks are assigned to some SVs.

Task $k_{i}$ sends its offloading information to all its neighbors to remove its selected SV from their permissible SVs list. Finally, this task is removed from $\mathbf{V}$ and the remaining tasks update their own $\mathcal{U}^{\text {cap }}, \mathcal{G}(\mathbf{V}, \mathbf{E}), \mathcal{T}(m, n)$. The algorithm continues to next round iteratively. If none of the SVs are selected for $k_{i}$, that means the algorithm could not find a complete solution and it starts the next iteration with another task. Note that only one task is processed within one round through the algorithm.

Since there are multiple restrictions and constraints in DROP, including privacy and accessibility restrictions, and SVs capacities, we devise the next algorithm based on a proactive renew rule to enhance performance in finding efficient solutions.

\subsection{Distributed Renew Algorithm}

In this subsection, we present the distributed renew algorithm based on a proactive renew rule to increase the probability of finding a complete assignment.

The distributed renew algorithm, given in Algorithm 3, is called with $\mathcal{S}=\emptyset$. It first collects tasks with the minimum number of permissible SVs. If any of these tasks has a conflict with another task in the set, the task with the minimum degree is selected. In case of a tie, the task is selected randomly. Otherwise, if no edge exists

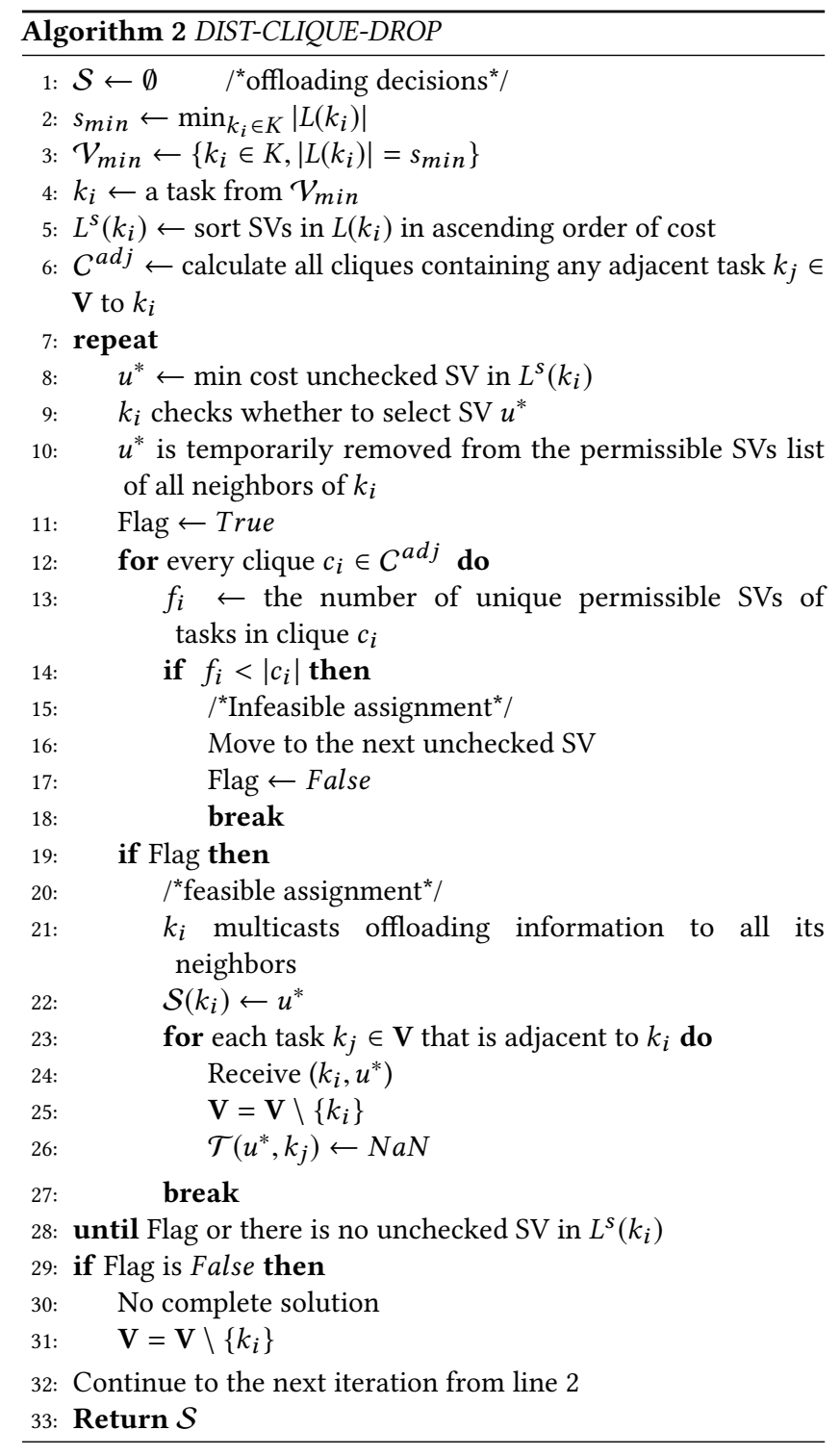

among the tasks in $\mathcal{V}_{\min }$, a task with the minimum degree (not including tasks with degree of zero) is selected. The selected task chooses an SV from its permissible SVs list which has the minimum overhead cost. Then, it multicasts the offloading information to all its neighbors in order to remove the selected SV from their own permissible SVs list. Finally, the remaining tasks update their $\mathcal{U}^{\text {cap }}, \mathcal{G}(\mathbf{V}, \mathbf{E})$ accordingly. This procedure continues iteratively.

With defining these priorities for offloading, the above procedure can largely reduce the chance of finding infeasible solutions. To further boost the probability of finding a complete assignment, we then design a proactive renew rule as shown in Algorithm 4. In doing so, a task $k_{i} \in \mathbf{V}$ is selected randomly and the first SV in $L^{s}\left(k_{i}\right)$ will be selected for offloading of task $k_{i}$. If a complete solution is not obtained by DIST-RENEW-DROP, the proactive renew rule updates the offloading decision of task $k_{i}$ to the next SV in $L^{s}\left(k_{i}\right)$. After checking all permissible SVs of a task if the proactive renew rule 

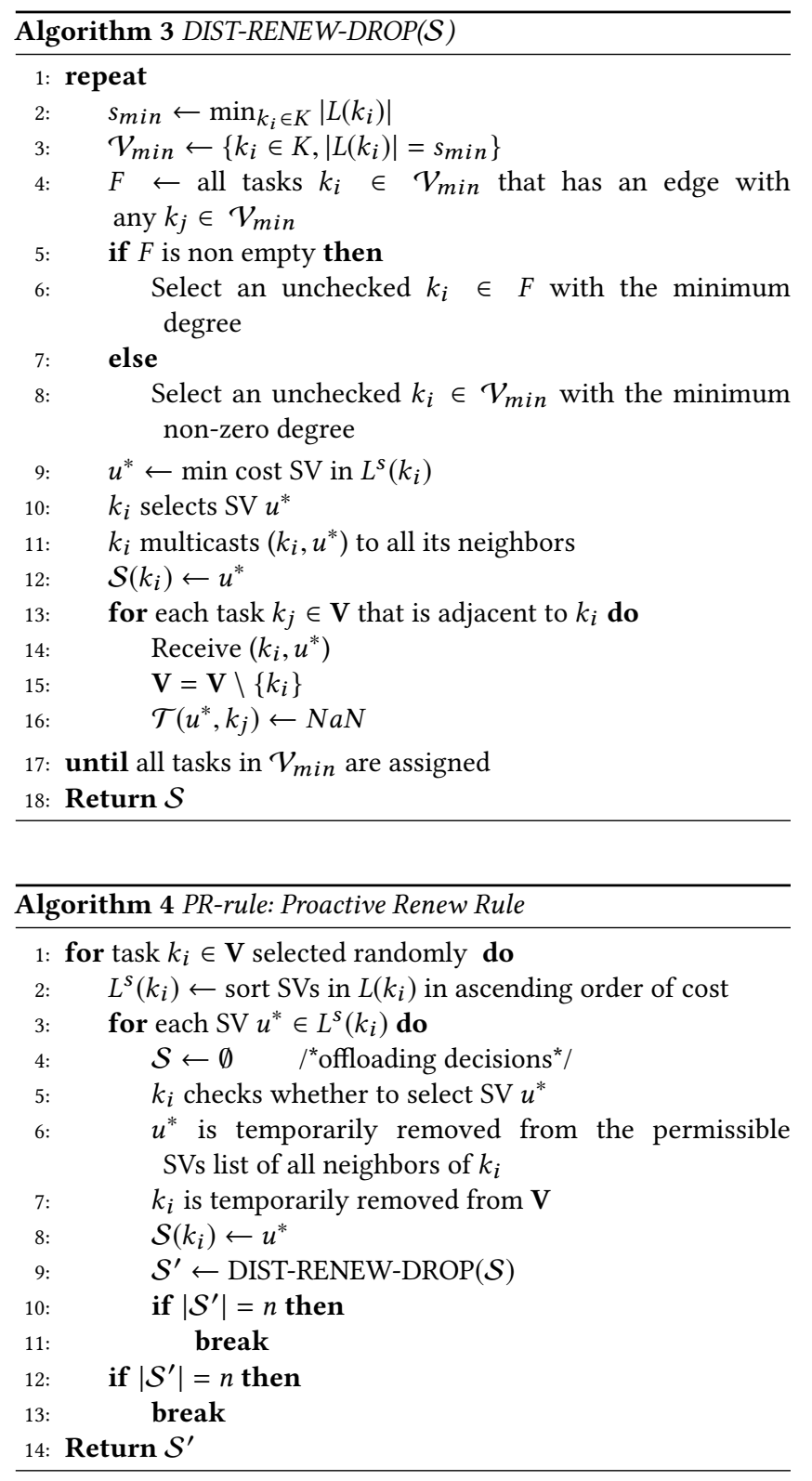

cannot find a complete solution, it halts and moves to the next task that is randomly selected. Note that the proactive renew rule only applies when Algorithm 3 cannot achieve a complete solution. Our experimental results demonstrate that the proactive renew rule greatly improves the probability of finding a complete assignment.

\section{EXPERIMENTAL RESULTS}

In this section, we comprehensively evaluate the performance of our proposed algorithms from three aspects: finding a complete assignment, the overhead cost, and execution time. For a benchmark, we obtain the optimal solution, called DROP-OPTIMAL, using IBM ILOG CPLEX Optimization Studio for Academics Initiative (Python API). The proposed algorithms and DROP-OPTIMAL are
Table 1: Parameters

\begin{tabular}{ccl}
\hline Parameter & Value & \multicolumn{1}{c}{ Description } \\
\hline$\mu_{c}$ & $40 \times 10^{3}$ Megacycles & mean of CPU cycles of SVs \\
$\mu_{s}$ & $5 \mathrm{MB}$ & mean of memory size of SVs \\
$\mu_{b}$ & $5.0 \mathrm{MHz}$ & mean of transmission bandwidth of SVs \\
$\mu_{f}$ & $5.0 \mathrm{GHz}$ & mean of CPU frequency of SVs \\
$\mu_{d}$ & $100 \mathrm{~m}$ & mean of distance between $u_{0}$ and other SVs \\
$\gamma$ & $1.0 \times 10^{-28}$ & effective switched capacitance of SV \\
$C_{i}$ & {$[1000-4000]$ Megacycles } & computational cycles of task $k_{i}$ \\
$D_{i}$ & {$[100-500] \mathrm{KB}$} & data size of task $k_{i}$ \\
$P_{0}$ & $0.1 \mathrm{Watts}$ & transmission power of $u_{0}$ \\
$h$ & 3.4 & path-loss factor \\
$N_{0}$ & $4 \times 10^{-15}$ Watts & background noise power \\
\hline
\end{tabular}

implemented in Python 3.6, and the experiments are conducted on $2.3 \mathrm{GHz}$ Intel Core i5 with $16 \mathrm{~GB}$ of RAM.

\subsection{Experimental Setup}

We consider a set of ten SVs are scattered across an area in which they can communicate with $u_{0}$. As a result, we consider the distance $d_{0 i}$ between $u_{0}$ and $u_{i}$ obeys Gaussian distribution with mean $\mu_{d}$ and standard deviation $\sigma_{d}=0.25 \mu_{d}$. Moreover, the CPU frequency of SVs obeys Gaussian distribution with mean $\mu_{f}$ and standard deviation $\sigma_{f}=0.30 \mu_{f}$.

For the wireless access, we set the transmission bandwidth $B_{i}$ obeys Gaussian distribution with mean $\mu_{b}$ and standard deviation $\sigma_{b}=0.30 \mu_{b}$. We consider diffrenet number of tasks $n=$ $\{20,30,40,50,60,70,80\}$ at $u_{0}$. For the computational tasks, similar to the previous studies $[16,19]$, we set $\left(C_{i}, D_{i}\right)$ of task $k_{i}$ uniformly selected from $[1000,2000,3000,4000]$ Megacycles and $[100,200,300,400,500] \mathrm{KB}$, respectively. To experiment with a set of heterogeneous SVs, CPU cycles $C_{i}^{c a p}$ and memory $D_{i}^{c a p}$ of $u_{i}$ obey Gaussian distribution with mean $\mu_{c}, \mu_{s}$ and standard deviation $\sigma_{c}=0.30 \mu_{c}, \sigma_{s}=0.30 \mu_{s}$, respectively. In addition, $e_{j}$ is calculated using $e_{j}=\gamma F_{j}^{2}$ [8], where $\gamma$ is the effective switched capacitance.

To enable tractable analysis and useful insights, we assume that the energy cost and delay cost are equally important for each tasks $k_{i}$, i.e., $\alpha_{i}=\beta_{i}=0.5$. The main parameters used in the experiments are summarized in Table 1 .

For the privacy restrictions, we setup a well-known random graph model, the Erdös-Rényi model [3], as the conflict graph $\mathcal{G}(\mathbf{V}, \mathbf{E})$. An Erdös-Rényi graph $(n, P)$ is a random graph constructed by connecting vertices randomly, where $n$ is number of tasks and any conflict (edge between any pair of tasks) has a probability of $P$. Based on the

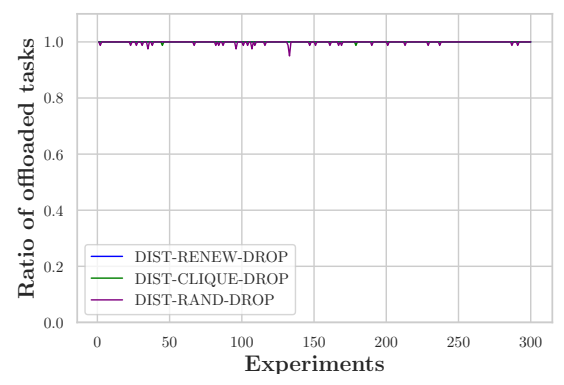

Figure 3: Complete assignment ratio 


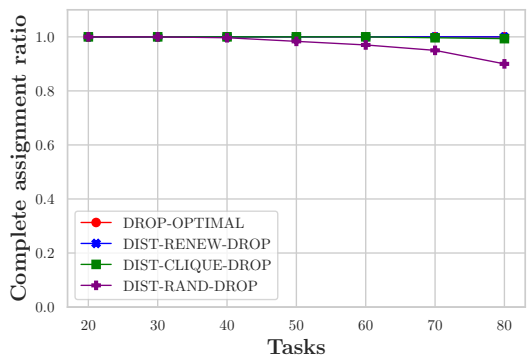

(a) different number of tasks $n$ $\left(P=0.12 ; P_{01}=0.4\right)$

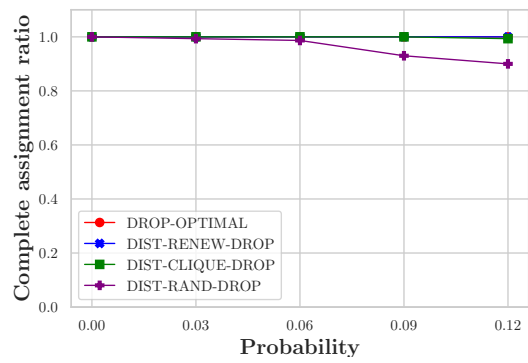

(b) different values of $P$ $\left(n=80 ; P_{01}=0.4\right)$

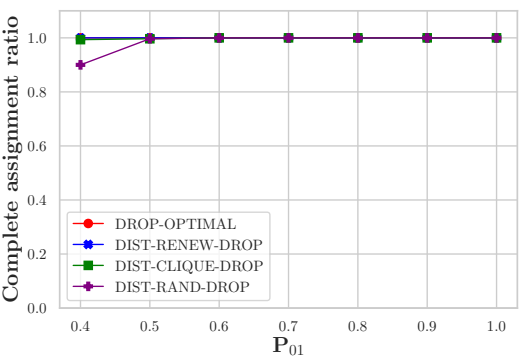

(c) different values of $P_{01}$ $(n=80 ; P=0.12)$

Figure 4: Complete Assignments: Sensitivity Analysis

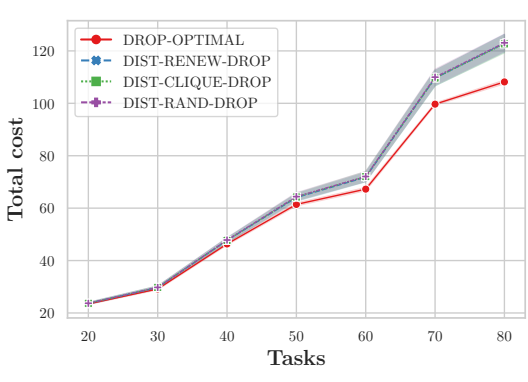

(a) different number of tasks $n$ $\left(P=0.12 ; P_{01}=0.8\right)$

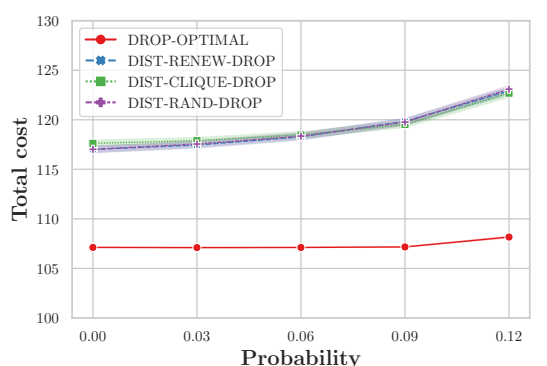

(b) different values of $P$ $\left(n=80 ; P_{01}=0.8\right)$

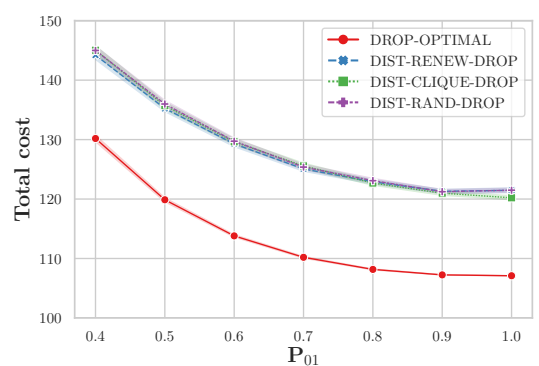

(c) different values of $P_{01}$ $(n=80 ; P=0.12)$

Figure 5: Overhead Cost: Sensitivity Analysis

value of $P \in[0,1]$, we can obtain a sparse or complex conflict graph. For the accessibility restrictions, the probability associated with SVs for each task (column) in matrix $\mathcal{T}$ is $P_{01}$ (the probability of generating 1 using the Bernoulli distribution). In our experiments, we study the performance of the proposed algorithms with different values of $n, P$, and $P_{01}$.

\subsection{Analysis of Results}

In this subsection, we evaluate the performance of the proposed algorithms in finding complete assignments, obtained overhead cost, and execution time.

We first analyze the assignments to find out the percentage of offloaded tasks in the obtained solutions of our algorithms. We fix $\left(n, P, P_{01}\right)=(80,0.12,0.4)$ in which both of the privacy and accessibility restrictions are constricted. As shown in Fig. 3, even though our proposed algorithms cannot always ensure complete assignments, the obtained results in 100 experiments show that the algorithms are able to achieve close to complete assignments, where at least $95.0 \%$ of tasks are offloaded.

To further verify the performance of the proposed algorithms in finding complete assignments, we perform sensitivity analysis on the number of tasks $n$, value of $P$, and $P_{01}$ (results are shown in Fig. 4). In each set of experiments, we fix the other two parameters. We present the complete assignment ratio defined as the number of experiments with complete assignments over the total number of experiments. Fig. 4a shows the performance of the algorithms with different number of tasks, considering $P=0.12$ and $P_{01}=0.4$. The complete assignment ratio is at least $90.0 \%$ by DIST-RANDDROP in 100 experiments. Fig. 4b shows the performance of the algorithms with different values of $P$, considering $n=80$ and $P_{01}=0.4$. The results show that the proposed algorithms are able to achieve a high complete assignment ratio as the probability of having conflicts increases. Fig. $4 \mathrm{c}$ presents the performance of the algorithms with different values of $P_{01}$, considering $n=80$ and $P=0.12$. Similarly, the proposed algorithms obtain high complete assignment ratio as $P_{01}$ (SVs accessibility) increases. Overall, DISTRENEW-DROP outperforms the other two proposed algorithms in terms of complete assignment ratio due to the fact that it executes a proactive renew rule once an unfeasible solution happens. DISTRAND-DROP, however, performs the worst compared to the other two algorithms. Since it does not consider an iterative rule for improving the probability of obtaining a complete assignment.

We then analyze the performance of the proposed algorithms in terms of the obtained overhead cost compared to the optimal cost obtained by DROP-OPTIMAL. As shown in Fig. 5, the proposed algorithms obtain near optimal solutions with respect to different values of $N, P$, and $P_{01}$. Specifically, the dashed lines represent the average cost obtained by the proposed algorithms, and the red line indicates the average optimal cost obtained by DROP-OPTIMAL. 


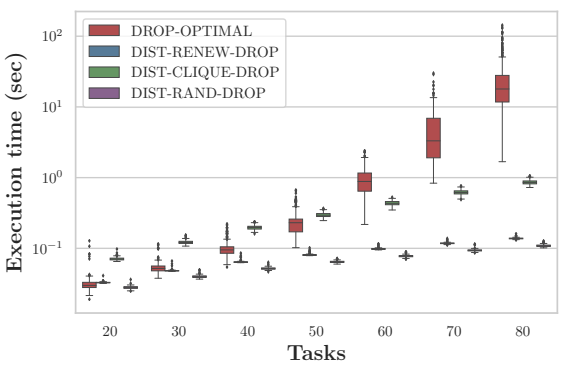

(a) different number of tasks $n$ $\left(P=0.12 ; P_{01}=0.8\right)$

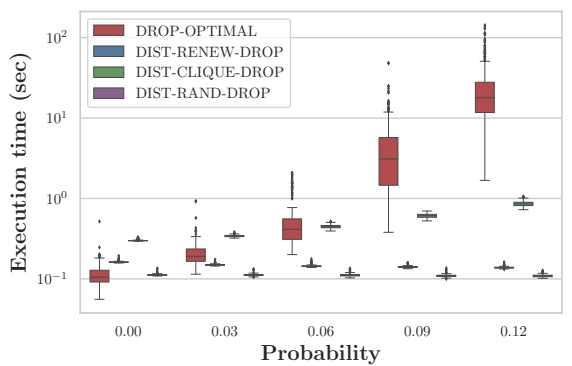

(b) different values of $P$ $\left(n=80 ; P_{01}=0.8\right)$

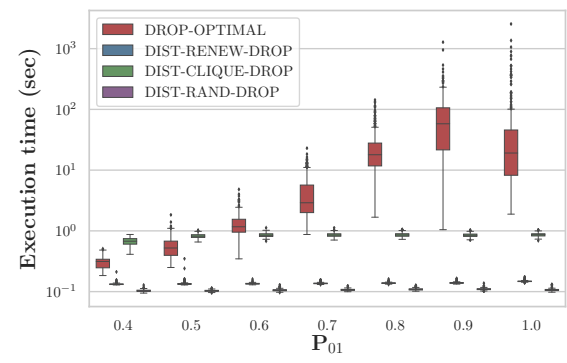

(c) different values of $P_{01}$ $(n=80 ; P=0.12)$

Figure 6: Execution Time: Sensitivity Analysis

Moreover, the standard deviation of the obtained solutions is presented in shaded error bands (semi-transparent areas). The results show that the the standard deviation is low and the average optimality gap remains small in all experiments. This is due to the fact that our proposed algorithms always allocate a proper SV with minimum cost to the selected task at each round in any experiment.

Finally, we verify the performance of the proposed algorithms in terms of execution time. Fig. 6a shows that the proposed algorithms obtain the results in a short amount of time and scale well as the number of tasks increases. Moreover, their execution time is stable in 100 experiments as the standard deviation is very low compared to the execution time of the optimal algorithm, DROPOPTIMAL, obtained by CPLEX. We also study the performance of algorithms with different values of $P$ and $P_{01}$. Again, Fig. $6 \mathrm{~b}$ and Fig. $6 \mathrm{c}$ show that our proposed algorithms are fast. The execution time by DROP-OPTIMAL can be very unstable with respect to the three parameters $N, P$, and $P_{01}$ due to its NP-hardness. It can only be the best choice for solving DROP when the problem size is very small, which is not practical.

From the above results, we conclude that the proposed algorithms are able to achieve complete assignments with a very high probability. In case that a complete assignment is not achieved, the proposed algorithms are still able to offload almost all tasks. Moreover, the proposed algorithms are scalable and obtain near optimal overhead cost for offloading in a short amount of time.

\section{CONCLUSION}

In this paper, we formulated the general Data pRotection Offloading Problem (DROP) in a network of capacitated SV-mounted cloudlets and proved its NP-hardness. We proposed three distributed iterative algorithms based on different updating rules. The experimental results demonstrate that the proposed algorithms are efficient in terms of overhead cost and execution time. In addition, they scale well as the system size grows. In our future work, we will study the impacts of SVs mobility on task offloading.

\section{ACKNOWLEDGMENTS}

This research was supported in part by NSF grant CNS-1755913.

\section{REFERENCES}

[1] Dixit Bhatta and Lena Mashayekhy. 2019. Generalized Cost-Aware Cloudlet Placement for Vehicular Edge Computing Systems. In Proceedings of the 11th IEEE International Conference on Cloud Computing Technology and Science (CloudCom). $1-8$.

[2] Xu Chen. 2014. Decentralized computation offloading game for mobile cloud computing. IEEE Transactions on Parallel and Distributed Systems 26, 4 (2014), 974-983.

[3] P Erdös and A Rényi. 1959. On random graphs. Publ. Math 6 (1959), 290-297.

[4] Jingyun Feng, Zhi Liu, Celimuge Wu, and Yusheng Ji. 2018. Mobile edge computing for the Internet of vehicles: Offloading framework and job scheduling. ieee vehicular technology magazine 14, 1 (2018), 28-36.

[5] Zhu Han, Yunan Gu, and Walid Saad. 2017. Matching theory for wireless networks. Springer.

[6] Dong Huang, Ping Wang, and Dusit Niyato. 2012. A dynamic offloading algorithm for mobile computing. IEEE Transactions on Wireless Communications 11, 6 (2012), 1991-1995.

[7] George Iosifidis, Lin Gao, Jianwei Huang, and Leandros Tassiulas. 2015. A doubleauction mechanism for mobile data-offloading markets. IEEE/ACM Transactions on Networking 23, 5 (2015), 1634-1647.

[8] Seongah Jeong, Osvaldo Simeone, and Joonhyuk Kang. 2017. Mobile edge computing via a UAV-mounted cloudlet: Optimization of bit allocation and path planning. IEEE Transactions on Vehicular Technology 67, 3 (2017), 2049-2063.

[9] David Jiang, Wang-Chiew Tan, Gang Chen, David Maier, Kian-Lee Tan, Beng Chin Ooi, and Hosagrahar Jagadish. 2014. Federation in Cloud Data Management: Challenges and Opportunities. IEEE Transactions on Knowledge and Data Engineering (2014), 1-14.

[10] Ewa Kubicka and Allen J Schwenk. 1989. An introduction to chromatic sums. In Proceedings of the 17th conference on ACM Annual Computer Science Conference. ACM, 39-45.

[11] Feng Luo, Chunxiao Jiang, Shui Yu, Jingjing Wang, Yipeng Li, and Yong Ren. 2017. Stability of cloud-based UAV systems supporting big data acquisition and processing. IEEE Transactions on Cloud Computing (2017).

[12] Weibin Ma, Xuanzhang Liu, and Lena Mashayekhy. 2019. A Strategic Game for Task Offloading among Capacitated UAV-Mounted Cloudlets. In Proceedings of the IEEE International Congress on Internet of Things (ICIOT). 61-68.

[13] Xiao Ma, Chuang Lin, Xudong Xiang, and Congjie Chen. 2015. Game-theoretic analysis of computation offloading for cloudlet-based mobile cloud computing. In Proceedings of the 18th ACM International Conference on Modeling, Analysis and Simulation of Wireless and Mobile Systems. ACM, 271-278.

[14] Lena Mashayekhy, Mark Nejad, and Daniel Grosu. 2019. A Trust-Aware Mechanism for Cloud Federation Formation. IEEE Transactions on Cloud Computing (in press) (2019).

[15] Lena Mashayekhy, Mahyar Movahed Nejad, and Daniel Grosu. 2014. A framework for data protection in cloud federations. In Proceedings of the 43rd International Conference on Parallel Processing. 283-290.

[16] Mohamed-Ayoub Messous, Hichem Sedjelmaci, Noureddin Houari, and SidiMohammed Senouci. 2017. Computation offloading game for an UAV network in mobile edge computing. In Proceedings of the IEEE International Conference on Communications (ICC). 1-6.

[17] Mohammad Mozaffari, Walid Saad, Mehdi Bennis, and Mérouane Debbah. 2016. Unmanned aerial vehicle with underlaid device-to-device communications: Performance and tradeoffs. IEEE Transactions on Wireless Communications 15, 6 (2016), 3949-3963. 
[18] Siani Pearson. 2009. Taking account of privacy when designing cloud computing services. In Proceedings of ICSE Workshop on Software Engineering Challenges of Cloud Computing. IEEE, 44-52.

[19] Lingjun $\mathrm{Pu}, \mathrm{Xu}$ Chen, Jingdong Xu, and Xiaoming Fu. 2016. D2D fogging: An energy-efficient and incentive-aware task offloading framework via networkassisted D2D collaboration. IEEE fournal on Selected Areas in Communications 34 12 (2016), 3887-3901.

[20] Theodore S Rappaport et al. 1996. Wireless communications: principles and practice. Vol. 2. prentice hall PTR New Jersey.

[21] Mahadev Satyanarayanan. 2017. The emergence of edge computing. Computer 50, 1 (2017), 30-39.

[22] Nafiseh Sharghivand, Farnaz Derakhshan, and Lena Mashayekhy. 2018. QoSAware Matching of Edge Computing Services to Internet of Things. In Proceedings of the 37th IEEE International Performance Computing and Communications Conference. 1-8.

[23] Neha Sharma, Madhavi Shamkuwar, and Inderjit Singh. 2019. The History, Present and Future with IoT. In Internet of Things and Big Data Analytics for Smart Generation. Springer, 27-51.

[24] Olena Skarlat, Vasileios Karagiannis, Thomas Rausch, Kevin Bachmann, and Stefan Schulte. 2018. A framework for optimization, service placement, and runtime operation in the fog. In Proceedings of the IEEE/ACM 11th International Conference on Utility and Cloud Computing (UCC). 164-173.
[25] Jyrki Wallenius, James S Dyer, Peter C Fishburn, Ralph E Steuer, Stanley Zionts, and Kalyanmoy Deb. 2008. Multiple criteria decision making, multiattribute utility theory: Recent accomplishments and what lies ahead. Management science 54, 7 (2008), 1336-1349.

[26] Rong Yu, Xumin Huang, Jiawen Kang, Jiefei Ding, Sabita Maharjan, Stein Gjessing, and Yan Zhang. 2015. Cooperative resource management in cloud-enabled vehicular networks. IEEE Transactions on Industrial Electronics 62, 12 (2015), 7938-7951.

[27] Daniel Zhang, Yue Ma, Chao Zheng, Yang Zhang, X Sharon Hu, and Dong Wang. 2018. Cooperative-competitive task allocation in edge computing for delaysensitive social sensing. In Proceedings of the IEEE/ACM Symposium on Edge Computing (SEC). 243-259.

[28] Hongli Zhang, Qiang Zhang, and Xiaojiang Du. 2015. Toward vehicle-assisted cloud computing for smartphones. IEEE Transactions on Vehicular Technology 64, 12 (2015), 5610-5618.

[29] Ke Zhang, Yuming Mao, Supeng Leng, Alexey Vinel, and Yan Zhang. 2016. Delay constrained offloading for mobile edge computing in cloud-enabled vehicular networks. In 2016 8th International Workshop on Resilient Networks Design and Modeling (RNDM). IEEE, 288-294.

[30] Zhenyu Zhou, Pengju Liu, Junhao Feng, Yan Zhang, Shahid Mumtaz, and Jonathan Rodriguez. 2019. Computation resource allocation and task assignment optimization in vehicular fog computing: A contract-matching approach. IEEE Transactions on Vehicular Technology 68, 4 (2019), 3113-3125. 